The Chinese Society of Theoretical and Applied Mechanics

Chinese Journal of Mechanics Press, Beijing, China

Allerton Press, INC., New York, U.S.A.

\title{
CONJUGATE MODEL FOR HEAT AND MASS TRANSFER OF POROUS WALL IN THE HIGH TEMPERATURE GAS FLOW*
}

\author{
A.F. Polyakov ${ }^{+} \quad$ D.L.Reviznikov ${ }^{++}$ \\ + (Institute of High Temperatures, Russian Academy of Sciences, Moscow, Russia) \\ ${ }^{++}$(Moscow Aviation Institute (Technical University), Moscow, Russia) \\ Shen Qing (沈 青) Tang Jinrong (唐锦荣) Wei Shuru (魏叔如) \\ (LHD, Institute of Mechanics, Chinese Academy of Science, Beijing 100080, China)
}

\begin{abstract}
Heat and mass transfer of a porous permeable wall in a high temperature gas dynamical flow is considered. Numerical simulation is conducted on the ground of the conjugate mathematical model which includes filtration and heat transfer equations in a porous body and boundary layer equations on its surface. Such an approach enables one to take into account complex interaction between heat and mass transfer in the gasdynamical flow and in the structure subjected to this flow. The main attention is given to the impact of the intraporous heat transfer intensity on the transpiration cooling efficiency.
\end{abstract}

KEY WORDS: heat and mass transfer, porous media, conjugate model, high temperature gas flow

\section{INTRODUCTION}

The most popular approach for describing heat transfer in porous media is the homogenous (one-temperature) model assuming equality of the porous frame and coolant temperatures throughout the wall thickness. One temperature model implies infinitely large intraporous heat transfer, i.e. the heat transfer between solid frame and gas-coolant flowing through it. However, the intraporous heat transfer intensity in real processes has finite value and the frame temperature usually differs from coolant temperature. To describe such a realistic situation properly a two-temperature model is used. The equations describing heat transfer in the porous frame and in the flowing coolant are written separately and the intensity of the intraporous heat transfer is used as the relation coupling the two equations. More detailed issues of the conjugate (two-temperature) model are discussed in Ref.[1 6]. The purpose of the presented paper is to investigate the problem on the basis of conjugate mathematical model when the processes of heat and mass transfer both in the porous wall and in the external flow are considered in a coupling way.

\section{MATHEMATICAL MODEL}

As a rule the effect of porous media temperature nonuniformity is studied by supposing

Received 13 July 2000, revised 22 February 2001

* The project supported by the National Natural Science Foundation of China (19889209) and Russian Foundation for Basic Research (97-02-16943) 
a given coolant flux through the wall. Under this circumstance, the qualitative picture is clear. The larger intraporous heat transfer coefficient is, the more intensive heat adsorption during the coolant filtration and the more substantial decrease of the frame temperature are. Therefore, the one-temperature model gives the lowest evaluation for the porous frame temperature.

However, the pressure difference between inner coolant space and external flow in many problems is a given value which determines the coolant flux. In this case the temperature dependence of coolant thermophysical properties becomes a significant factor for the determination of coolant flux.

Thus the problem has conjugate nature. On the one hand, coolant flux determines the intensity of heat energy adsorption and the rate of heat flux decrease due to pushing off the hot gas in the boundary layer. On the other hand, the coolant flux itself depends on temperature.

In order to take into consideration complex interrelated behavior of heat and mass transfer processes in the gas flow and in the structure being flown about, a conjugate mathematical model is used which includes the equations of boundary layer on the external surface, the equations of filtration and heat transfer in the porous wall and the corresponding conjugate conditions. The latter implies the equality of mass fluxes, temperatures and heat fluxes in different media. More comprehensive information about the conjugate model can be found in Ref.[7,8].

To eliminate effects of longitudinal coolant flux and wall temperature nonuniformity ${ }^{[7,8]}$ let us consider the flow around a flat plate with transpiration cooling (Fig.1). The equations describing filtration and heat transfer in the porous wall are as follows

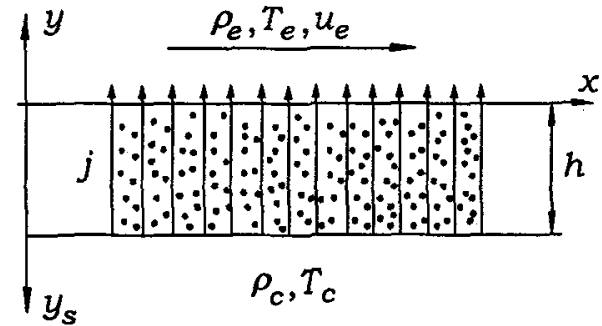

Fig.1 Scheme of the region investigated

$$
\begin{aligned}
& \rho_{f} \frac{\mathrm{d} p}{\mathrm{~d} y_{s}}=a \mu_{f} j+b j^{2} \\
& \frac{\mathrm{d}}{\mathrm{d} y_{s}}\left(\lambda_{s} \frac{\mathrm{d} T_{s}}{\mathrm{~d} y_{s}}\right)-Q_{v}=0 \\
& \frac{\mathrm{d}}{\mathrm{d} y_{s}}\left(\lambda_{f} \frac{\mathrm{d} T_{f}}{\mathrm{~d} y_{s}}\right)+c_{p_{f}} j \frac{\mathrm{d} T_{f}}{\mathrm{~d} y_{s}}+Q_{v}=0
\end{aligned}
$$

where $a, b$ the coefficients of the viscous and inertial resistance of the porous frame, $\rho_{f}, \mu_{f}$ the coolant density and dynamical viscosity, $j$ the absolute value of the specific coolant flux, $T$ is the temperature, $c_{p}$ the specific heat capacity, $\lambda$ the heat conductivity coefficient, index $s$ corresponds to the frame, $f$ is to the coolant. Equation (1) is the modified Darcy equation, expressing the relation between the pressure drop and the coolant flux, Eq.(2) describes heat transfer in the porous frame, Eq.(3) is in the gas-coolant. The boundary layer equations are traditional and thesefore not presented here. To describe turbulence in the boundary layer the algebraic Cebeci-Smith model ${ }^{[9]}$ is used.

The relation coupling Eqs.(2) and (3) is given by intensity of the intraporous heat transfer $Q_{v}$. To define it the volume heat transfer coefficient $\alpha_{v}$ is usually used. The reason for using the volume relative coefficient is the complexity in the determination of the 
intraporous surface taking part in the heat transfer process. The expression for $Q_{v}$ is as follows

$$
Q_{v}=\alpha_{v}\left(T_{s}-T_{f}\right)
$$

Experimental study of the intraporous heat transfer is a very compex problem. That is why scattering of different authors' results is enormoces ${ }^{[6]}$. Usually the coefficient $\alpha_{v}$ is presented in the following form

$$
\alpha_{v} \frac{l^{2}}{\lambda_{f}}=K(\operatorname{RePr})^{n}
$$

where $l$ the characteristic scale of the porous media, $R e, P r$ are the Reynolds and the Prandtl numbers for coolant. Some authors use porous diameter as a characteristic scale, others use the relation of the viscous and inertial resistance coefficients. Coefficients $K$ and $n$ are different in different works, so the dispersion of $\alpha_{v}$ calculated with (4), may reach one order. Therefore, it is important to study the impact of the above mentioned dispersion on the porous frame temperature field.

Finite difference iteration algorithms are used to solve the equation set. The boundary layer equations transformed to variables of the Dorodnitsyn-Lees type are approximated using implicit schemes. The conjugate conditions on the interface are realized with the successive approximations.

\section{RESULTS ANALYSIS}

Let us take input data featured for gas turbine blades of the envelope type ${ }^{[10]}$. The stagnation pressure and temperature in the external flow are $p_{0}=1.65 \mathrm{MPa}$ and $T_{0}=$ $1223 \mathrm{~K}$. The coolant temperature is $373 \mathrm{~K}$. Resistance coefficients of the porous material are taken from [6]: $a=1.25 * 10^{10} \Phi^{-5.3} \mathrm{~m}^{-2} ; b=2 . * 10^{4} \Phi^{-6} \mathrm{~m}^{-1}$. Here $\Phi$ is the value of the material porosity, $\Phi=0.5$. The heat conductivity coefficient $\lambda_{s}=10 \mathrm{Wt} / \mathrm{mK}$, the wall thickness $h=0.001 \mathrm{~m}$. The properties of the external flow gas and of the coolant are similar to air properties. Range of internal pressure variation provides that blow efficiency parameter $b_{t}=\frac{j}{\rho_{0} u_{0}} \frac{1}{S t^{*}}\left(S t^{*}\right.$ is Stanton number for impermeable surface, $\rho_{0}, u_{0}$ are the density and velocity of internal flow) does not exceed 2.

When the two-temperature model is used, some problems related to the setting of boundary conditions appear.

On the external hot surface the conjugate conditions are assigned to express the equality of the mass fluxes, temperatures and heat fluxes in gas and porous media. Here we face two questions. The first one is which temperature we should use as a wall temperature and the second is how to take into consideration coolant heat conductivity in Eq.(3). It is necessary to specify boundary condition for this equation correctly. Comprehensive study of these questions can be found in Ref.[4] where analytical solutions of the conjugate problem in some cases are given. In the present work the analysis of coolant heat conductivity contribution is performed for the above mentioned input data. Along with the common conjugate condition for solid phase $\left.\lambda_{s} \frac{\partial T_{s}}{\partial y_{s}}\right|_{y_{s}=0}=-q_{w}$ the similar expression $\left.\lambda_{f} \frac{\partial T_{f}}{\partial y_{s}}\right|_{y_{s}=0}=-q_{w}$ is used as the boundary condition for (3). Here $q_{w}$ is the specific heat flux from the hot gas. The results of the numerical simulation are shown in Fig.2. Here the temperature profiles for the porous frame (solid curves) and coolant (broken curves) are presented. The curves obtained taking 
into account coolant heat conductivity are indicated by markers. The intraporous heat transfer is calculated here using (4) with the coefficients $K=0.004, n=1$. As a characteristic intraporous scale relation $b / a$ is taken. Curves 1 and 2 correspond to different pressure drops between the inner space and the external flow $\left(1-p_{c} / p_{e}=1.0075,2-p_{c} / p_{e}=\right.$ 1.0175 ), curve 3 - to smaller frame heat conductivity $\left(\lambda_{s}=1 \mathrm{~W} / \mathrm{m} \cdot \mathrm{K}, p_{c} / p_{e}=1.0075\right)$. One can see that depending on determinative parameters of the problem the coolant temperature at the outlet from the porous wall may exceed the frame temperature and may be lower than $T_{s}$. The frame temperature itself depends on coolant heat conductivity in (3) quite weakly. So we can further neglect this factor. Therewith we accept the frame temperature as the external surface temperature.

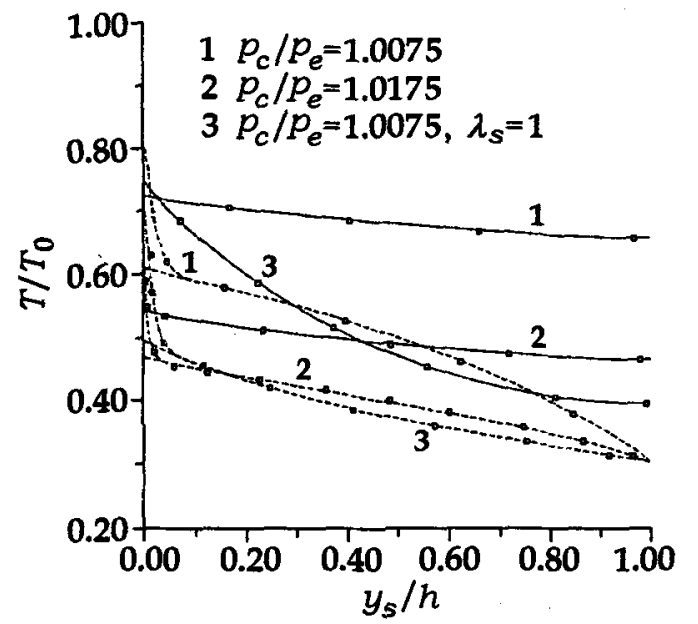

Fig.2 Impact of the coolant heat conductivity on the porous wall temperature field

Let us consider internal boundary conditions $\left(y_{s}=h\right)$ for heat transfer Eqs.(2), (3). The boundary condition for (2) (frame temperature) is quite obvious. Heat energy delivered to internal surface via the frame is consumed for heating gas in the coolant space and coolant at the direct entrance into the porous wall

$$
\lambda_{s} \frac{\mathrm{d} T_{s}}{\mathrm{~d} y_{s}}=-c_{p_{f}} j\left(T_{f}-T_{c}\right)-\alpha^{\prime}\left(T_{s}-T_{c}\right)
$$

In the case of weak heat transfer with the inner space $\left(\alpha^{\prime}=0\right)$, the regime of the asymptotic exhaust is realized. The problem of second boundary condition setting is more difficult. It is necessary to describe the coolant heating at the porous structure entry. Here the small-scale convective heat transfer appears in the porous vicinity ${ }^{[6]}$. This is the reason why using the traditional heat flux continuity condition is incorrect. Probably the most reasonable approach to set the second boundary condition is using the heat transfer coefficient $\alpha^{\prime \prime}$ so that the expression for the coolant temperature is

$$
\frac{T_{f}-T_{c}}{T_{s}-T_{c}}=\frac{\alpha^{\prime \prime}}{c_{p_{f}} j}=\omega
$$

However, it is very difficult to determine the concrete value of the parameter $\alpha^{\prime \prime}$ which is realized in the process investigated. If we neglect the heat transfer due to coolant heat conductivity we can separate two limiting cases: there is no coolant heating at the porous structure entry $(\omega=0)$ and the coolant temperature is equal to the frame temperature $(\omega=1)$. Thus in the two-temperature model along with intraporous heat transfer coefficient dispersion we have uncertainty in setting the parameter $\omega$. That is why it is necessary to study the impact of this parameter on the porous wall temperature field.

The results of parametric analysis are presented in Figs.3 and 4. Here the temperature distributions for the porous frame (solid curves) and for the coolant (broken curves) over the wall thickness are shown. Curves 1 and 3 correspond to limiting values of the parameter 
$\omega(\omega=0$ and $\omega=1)$, and curve 2 to $\omega=0.5$. The intraporous heat transfer coefficient is calculated by (4) $(K=0.004, n=1)$. The curves marked by quadrates correspond to the one-temperature model. If the pressure drop between the internal coolant space and the external flow is relatively high (Fig. $3, p_{c} / p_{e}=1.0175$ ) the coolant flux is determined par excellent by this drop and the change of $\omega$ affects the frame temperature quite weakly. All the curves $1 \sim 3$ lie above the one temperature model curve. This agrees well with the case of the given coolant flux (the one-temperature model corresponds to the limiting case of the infinitely large intraporous heat transfer coefficient). When pressure drop decreases (Fig.4, $p_{c} / p_{e}=1.0075$ ) the picture is changed. Here the temperature dependence of coolant flux becomes significant. As a result curves 1 and 2 lie below the one-temperature model curve. Thus conjugate character of the processes gives some singularities in the results of the parametric analysis.

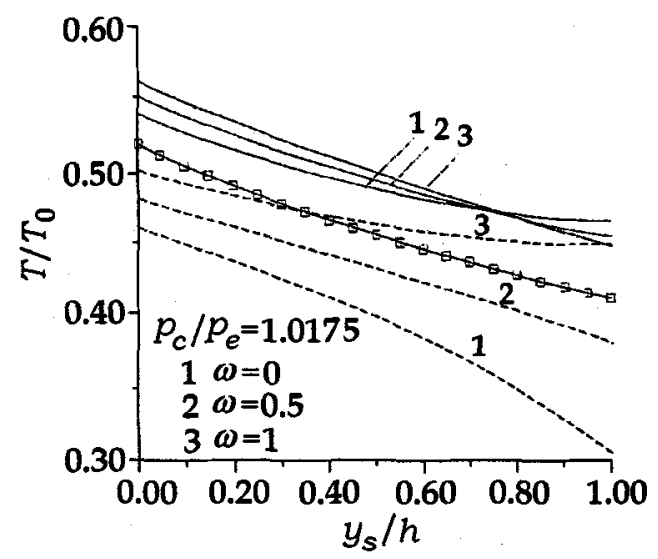

Fig.3 Distribution of porous frame and coolant temperatures over the wall thickness at different values of coefficient $\alpha^{\prime \prime}$. The case of the high pressure drop

Furthermore we consider impact of the intraporous heat transfer coefficient on the frame temperature. The results of the numerical simulation are shown in the Fig.5. The case of absence of coolant heating at the porous structure entry is considered $(\omega=0)$. The results presented are obtained using the expression (4) for $\alpha_{v}$. For solid curves $K=0.004, n=1$, broken curves correspond to increase of $\alpha_{v}(K=0.02, n=1)$. Curves $1 \sim 3$ are obtained for different values of the pressure drop. If this value is relatively high (curve $3, p_{c} / p_{e}=1.0175$ ) increase of the

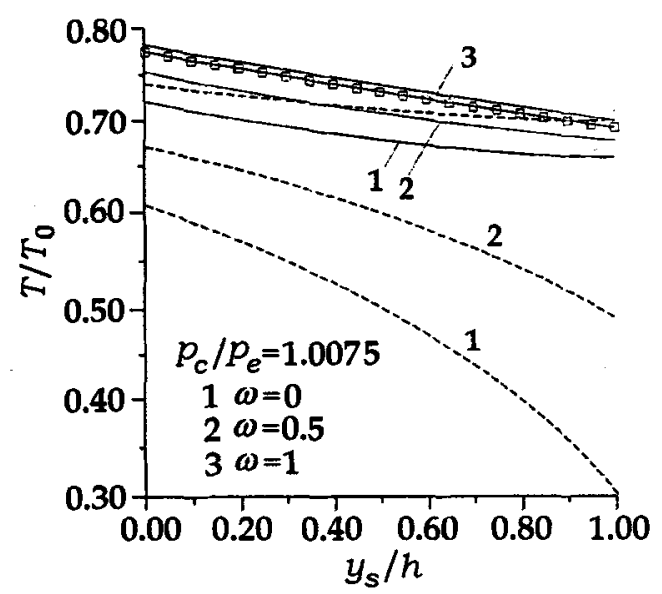

Fig.4 Distribution of porous frame and coolant temperatures over the wall thickness at different values of coefficient $\alpha^{\prime \prime}$. The case of the low pressure drop

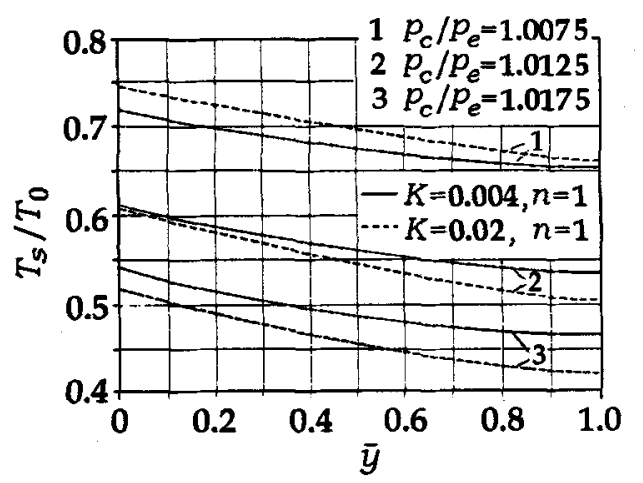

Fig.5 Impact of the intraporous heat transfer coefficient on the porous frame temperature 
intraporous heat transfer coefficient is accompanied by more intensive heat adsorption and the frame temperature decreases. At the smaller pressure drop (curve $2, p_{c} / p_{e}=1.0125$ ) the temperature dependence of the coolant flux begins to affect the process. Increase of $\alpha_{v}$ is accompanied by more intensive coolant heating. Meanwhile its thermophysical properties are changed (the density becomes lower and the viscosity increases). It results from the coolant flux reduce at the given pressure drop. That in its turn brings the descent of the pushing effect in the boundary layer and consequently the heat flux from the hot gas increases. As a result the tendency of frame temperature increase appears. This tendency becomes dominant at the low pressure drop (curves $1, p_{c} / p_{e}=1.0075$ ). Here the broken curve corresponding to the high intraporous heat transfer coefficient lies above the solid curve. Thus, the intensification of the intraporous heat transfer depending on the pressure drop between the internal coolant space and the external flow may lead to either increase or decrease of the cooling efficiency.

\section{REFERENCES}

1 Sahraoui M, Kaviany M. Slip and no-slip temperature boundary conditions at the interface of porous plain media: convection. Int J Heat Mass Transfer, 1994, 37(6): 1029 1044

2 Beavers G S, Joseph D D. Boundary conditions at a naturally permeable wall. $J$ Fluid Mech, 1967, 30: 197 207

3 Polezhaev Y V, Polyakov A F, Potsepkin V M, et al. Thermal regimes of the porous wall at the transpiration cooling. High Temperature (Engl Transh), 1997, 35(1): 83 89

4 Leont'ev A I, Polyakov A F. Convective heat transfer conditions on the porous permeable wall surface. Izv Ross Akad Nauk Energ, 1998, (6): 120 143 (in Russian)

5 Eckert $\mathrm{E}$ R G, Cho H H. Transition from transpiration to film cooling. Int $J$ Heat Mass Transfer, 1994, 37(1): 3 8

6 Polyaev V M, Maiorov V A, Vasil'ev L V. Hydrodynamics and Heat Transfer of Porous Elements of Structures of Flying Vehicles. Moscow: Machinery, 1988 (in Russian)

7 Polyakov A F, Reviznikov D L. Numerical simulation of conjugate heat transfer under conditions of transpiration cooling of cylindrical front edge. High Temperature (Engl Transl), 1998, 36(4): $593 \sim 599$

8 Polyakov A F, Reviznikov D L. Numerical simulation of conjugate heat and mass transfer under conditions of convection-and-curtain transpiration cooling. High Temperature (Engl Transl), 1999, 37(4): 393 400

9 Cebeci T, Bradshaw P. Physical and Computational Aspects of Convective Heat Transfer. New York: Springer, 1984

10 Kuz'min R B, Polezhaev Y V, Polyakov A F, et al. Numerical simulation of conjugate heat transfer under flow around gas turbine blades. High Temperature (Engl Transl), 1995, 33(4): $602 \sim 609$ 